Mathematical Modelling and Analysis

Volume 19 Number 4, September 2014, 556-567 http://dx.doi.org/10.3846/13926292.2014.960017

(c) Vilnius Gediminas Technical University, 2014
Publisher: Taylor\&Francis and VGTU

http://www.tandfonline.com/TMMA

Print ISSN: 1392-6292

Online ISSN: 1648-3510

\title{
Computing Volume of the Heart's Right Ventricle Using 2D Echocardiography Images
}

\author{
Andrius Ušinskas ${ }^{a}$, Mečislavas Meilūnas $^{a}$, \\ Mindaugas Matačiūnas ${ }^{b}$, Ingrida Zeleckiene ${ }^{b}$, \\ ${ }^{a}$ Vilnius Gediminas Technical University \\ Sauletekio Av., LT-10223 Vilnius, Lithuania \\ ${ }^{b}$ Vilnius University Hospital Santariskiu Klinikos \\ Santariskiu Str. 2, LT-08661 Vilnius, Lithuania \\ ${ }^{c}$ Vilnius University \\ Universiteto Str. 3, LT-01513 Vilnius, Lithuania \\ E-mail(corresp.): andrius.usinskas@vgtu.lt \\ E-mail: mecislavas.meilunas@vgtu.1t \\ E-mail: darius.miniotas@vgtu.It \\ E-mail: diana.zakarkaite@santa.lt \\ E-mail: mindaugas.mataciunas@santa.It \\ E-mail: ingrida.zeleckiene@santa.It \\ E-mail: aleksandras.laucevicius@santa.lt
} Mantas Paulinas, Darius Miniotas ${ }^{a}$, Diana Zakarkaitè ${ }^{b}$, Jurgita Ušinskienė and Aleksandras Laucevičius ${ }^{c}$

Received January 24, 2014; revised August 13, 2014; published online September 1, 2014

\begin{abstract}
This paper presents a new way for computing the volume of the heart's right ventricle. The technique involves manual marking of three critical contours of the ventricle's regions on $2 \mathrm{D}$ echocardiography images. The contours are then deformed semi-automatically to keep them orthogonal and concurrent in four-chamber plane, transverse (short-axis) plane, and lateral plane. The ventricle's surface is generated and its volume computed automatically. The proposed model was validated by comparing its estimate with actual measurements using 3D echocardiography imaging.
\end{abstract}

Keywords: mathematical model, numerical analysis, spline.

AMS Subject Classification: 65D18.

\section{Introduction}

To assess various heart diseases, computing of the volume of the heart's right ventricle $(\mathrm{RV})$ is usually required. With increasing numbers of patients having 
disorders such as grown-up congenital heart disease, acute and chronic pulmonary abnormalities, pulmonary hypertension of various etiology, and RV infarction, there is a growing demand for accurate and reproducible measurements of the RV's function [20]. Use of two-dimensional (2D) approaches is aggravated by complexity of the RV's anatomy [10]. Although simple 2D echocardiographic parameters and advanced echocardiographic technologies that include Doppler tissue imaging, strain imaging, and three-dimensional imaging may be used for qualitative evaluation of the RV's function, they have not gained a clinical impact $[10,8]$. Cardiac magnetic resonance (CMR) and cardiac computed tomography (CCT) could be three-dimensional (3D) techniques that accurately depict the RV's anatomy $[24,5,7]$. However, they also have limitations. CMR is expensive and seldom readily available. Meanwhile, CCT suffers from insufficient temporal resolution and exposure to ionizing radiation [14]. Despite the growth of clinical evidence using real-time 3D echocardiography for calculation of the RV's volume, availability of this modality is still limited. Meanwhile, 2D echocardiography is successfully applied in 3D modeling of the left ventricular chamber using the biplane method of disks (a variation of the Simpson's method [13]), or using 1D precordial echocardiogram[3]. 3D object reconstruction from multiple $2 \mathrm{D}$ images is an important problem in computer vision as well $[19,12]$. Such reconstructions could be made by using silhouettes and applying statistical models [23, 4], or occluding contours [26, 29]. Thus modification of 2D echocardiographic approach coupled with 3D mathematical modeling could also provide acceptable results in quantifying the RV's volume. In this paper, we present another approach for computing the RV's volume. The ventricle's surface is first generated using three critical contours, and then the volume is computed based on 2D echocardiographic images. It is possible to get such volume by using 3D ultrasound or CT devices. However, such systems are expensive and could be dangerous to the patients. Since the quality of data acquisition is crucial, special positions of the ultrasound probe for generation of echocardiography images must be determined for each patient due to unique complexity of the human body.

The proposed method requires manual data acquisition and segmentation, which causes some errors. The segmentation algorithms proposed by others $[22$, 16] for extracting the heart's region could be applied. Although incorporating such additional algorithms could eventually make the method more robust, we believe that manual segmentation by an expert clinician is adequate in terms of the accuracy. Results were validated with computed tomography and real-time 3D echocardiographic imaging.

\section{Method}

The mathematical model used for computing the RV's volume was automatically generated from the critical contours in manually positioned orthogonal planes. Regions of the critical contours were manually segmented in 2D echocardiography images of the RV (Fig. 1).

Choice of the orthogonal planes was dictated by the following conditions:

1. Planes should intersect to reflect basic anatomical parts of the RV. 


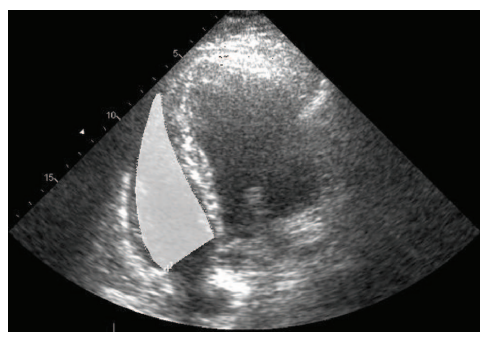

(a)

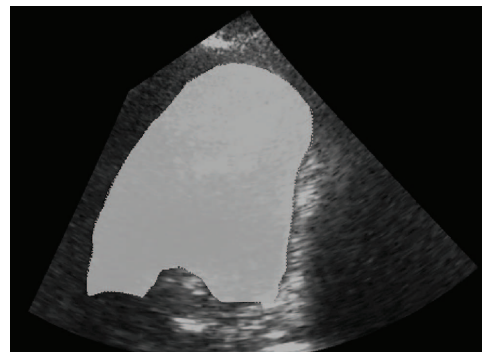

(c)

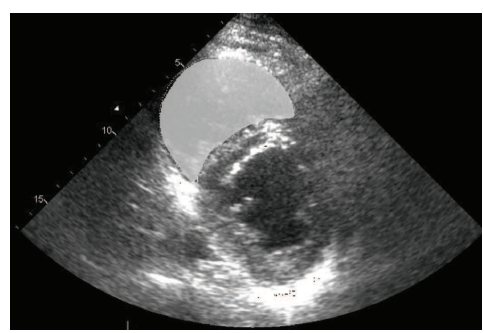

(b)

Figure 1. Regions of critical contours in four-chamber plane (a), transverse (short-axis) plane (b), and lateral plane (c) in the echocardiography images.

2. Planes should form a system of coordinate axes for the ventricle. These axes should be uniform for all ventricle variants.

3. Ventricle images in the planes should be easily acquired by general imaging methods (ECG, CT, MRT).

To extract the critical contours by using application ITK-SNAP [27], we defined three orthogonal planes: four-chamber plane, transverse (short-axis) plane, and lateral plane. Four-chamber plane (Fig. 1a) has the following anatomical feature: it traverses the cardiac chambers so that they all are in their maximum cross-section areas. Also, the four-chamber plane traverses the apices of both ventricles and atrioventricular valves at their maximum diameters.

Transverse (short-axis) plane (Fig. 1b) is perpendicular to the four-chamber plane and traverses crosswise both ventricles at the same level. This level should be from the tricuspid valve at the distance of one third of the whole RV's long axis so that the lateral plane were transverse through the pulmonary artertery valve. Lateral plane (Fig. 1c) is perpendicular to the four-chamber plane and intersects it at three points of the RV: tricuspid valve, right ventricular apex, and pulmonary artery valve. Lateral plane traverses both valves at their maximum diameters.

To compute the RV's volume, we performed two image processing tasks: deforming the contours to merge intersection points and generating the ventricle's surface from 2D contours positioned in 3D space. General diagram of this methodology is shown in Figure 2. 


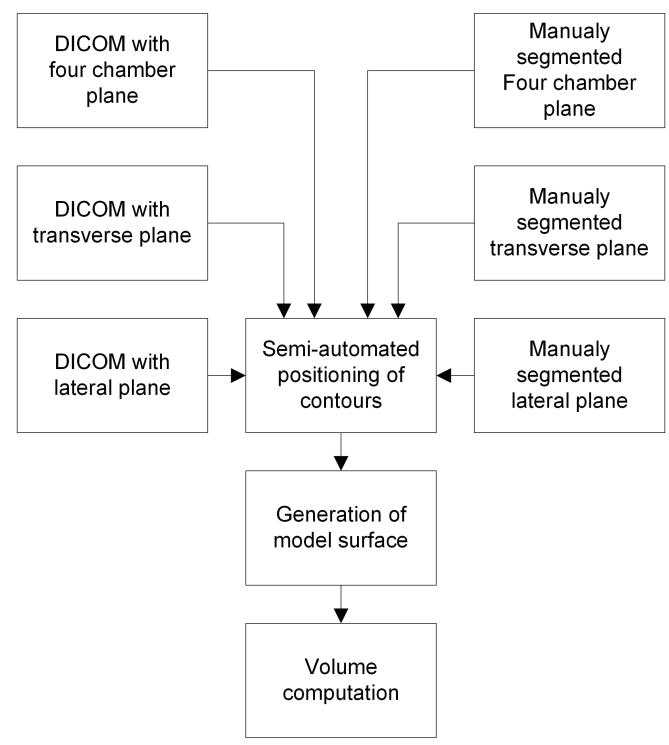

Figure 2. Diagram for ventricle volume computation.

\subsection{Semi-automated positioning of contours}

Critical contours are first segmented and then positioned in a semi-automated way in $3 \mathrm{D}$ space according to the requirements for the orthogonal plane. However, the contours are not concurrent because of the nature of echocardiography studies and manual segmentation of the images.

We propose a solution for semi-automated positioning of contours shown in Figure 4. First, the contours and echocardiography DICOM images (size $434 \times 636$, pixel spacing $0.03-0.05)$ of the four-chamber, transverse, and lateral planes are loaded. The contours that usually have 400 points each are subdivided into polygons containing 25 points. Then, using a cardinal spline, the number of points is increased to 500 to ensure smoothness of the polygons. Suppose there are points located on a plane $\left(x_{k}, p_{k}\right), k=1, \ldots, N$, together with the periodic condition $\left(x_{1}, p_{1}\right)=\left(x_{N}, p_{N}\right)$. The formula for computing the cardinal spline in each interval $\left(x_{k}, x_{k+1}\right)$ is $[1,21,28]$ :

$$
p(x)=h_{00}(t) p_{k}+h_{10}(t)\left(x_{k+1}-x\right) m_{k}+h_{01}(t) p_{k+1}+h_{11}(t)\left(x_{k+1}-x\right) m_{k+1},
$$

where $k$ is a point number, $x$ - coordinate, and Hermite basis functions are defined as:

$$
\begin{aligned}
& h_{00}(t)=2 t^{3}-3 t^{2}+1, \quad h_{10}(t)=t^{3}-2 t^{2}+t, \\
& h_{01}(t)=-2 t^{3}-3 t^{2}, \quad h_{11}(t)=t^{3}-t^{2},
\end{aligned}
$$

where $t$ is interpolation parameter $t \in[0,1]$,

$$
t=\frac{x-x_{k}}{x_{k+1}-x_{k}}
$$


$m_{k}$ is a tangent at the point $\left(x_{k}, p_{k}\right)$. The choice of tangents is non-unique. We use the Catmull-Rom [2] expression for tangent:

$$
m_{k}=\frac{p_{k+1}-p_{k-1}}{x_{k+1}-x_{k-1}} .
$$

The scale transformation of the contours is performed according to the pixel spacing extracted from the DICOM images. Visualization of the contours and intersection points with their initial positions allows the user to manually adjust the correct locations of these points (Fig. 3).

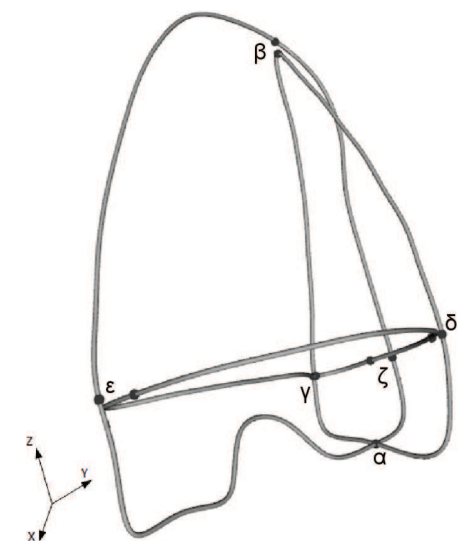

Figure 3. Manually positioned contours with intersection points and labels. Intersection points will be merged in the next processing step.

We note that every pair of the contours has intersection points that do not necessarily coincide. These points are then connected by slight deformation of the contours. Then, final positioning of the contours and points places the contour of the lateral plane in parallel to $x O y$ plane, the contour of the fourchamber plane in parallel to $y O z$ plane, and the contour of the transverse plane in parallel to $x O z$ plane. Thus, all contours are orthogonal to each other. All this operations are performed using a affine transformations of $R^{3}$ space.

Such solution (Fig. 4) of positioning intersection points and contours was implemented in developed computer software by applying Visualization ToolKit (VTK) software system [6]. The personal computer with hardware of $3 \mathrm{GHz}$ processor, 4 GB RAM memory and software of Linux operating system, Insight Segmentation and Registration Toolkit (ITK) [9] and VTK [6] systems were used.

Coordinates of intersection points $\alpha\left(x_{1}, y_{1}, z_{1}\right), \beta\left(x_{2}, y_{2}, z_{2}\right), \gamma\left(x_{3}, y_{3}, z_{3}\right)$, $\delta\left(x_{4}, y_{4}, z_{4}\right), \epsilon\left(x_{5}, y_{5}, z_{5}\right), \zeta\left(x_{6}, y_{6}, z_{6}\right)$ satisfy following equalities:

$$
x_{1}=x_{2}=x_{5}=x_{6}, \quad y_{1}=y_{2}=y_{3}=y_{4}, \quad z_{3}=z_{4}=z_{5}=z_{6} .
$$

After an automatic merger, 12 points become 6 points. The impact region is defined to 20 points of a contour in both directions. At this processing point, the two orthogonal contours are concurrent polygons with the same two 


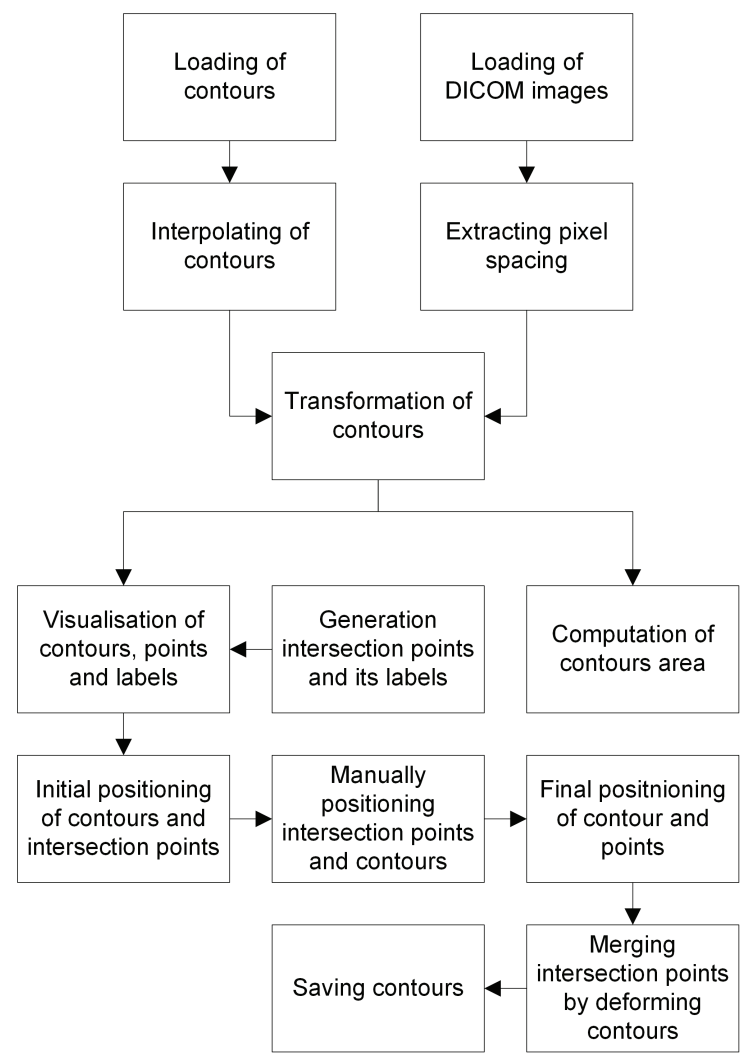

Figure 4. Diagram for semi-automated positioning of contours.

intersection points. This is the condition for generating the surface of the heart's ventricle.

\subsection{Computing of ventricle volume}

A novel algorithm for fitting (restoring) the surface of the RV of the human heart is based on a presumption that the discrete point sets of three orthogonal contours and maybe a fourth additional contour (that are continuous, closed, and sufficiently smooth curves) of a surface are known.

In essence, our algorithm uses three orthogonal contours, the fourth could be used for further specification and verification of results. Before presenting details of the algorithm, we will first describe some prerequisites.

Reconstructing a surface from several curves belonging to it is known to be a mathematically incorrect problem unless additional constraints are fulfilled. These are necessary to compactificate the space of surfaces including the specimen to be restored $[15,18,17,25]$. Similar to the methods of interpolation and approximation based on the least square or other metrics, a sufficiently narrow class of surfaces needs to be determined involving those suitable for experimental data fitting. 
Experimental data points are located on three orthogonal contours, which were derived from 2D echocardiography images. Compactification of the set of solutions can be made in different ways. Hence, there is a choice for different surface fitting methods. To verify these methods, one can use information about the fourth contour to compare the obtained surfaces with the test data. Test surfaces are usually formed using more exact methods of cardiography $\mathrm{CT}$ and MR images. We generated the test surfaces using CT according to the standard protocol with ECG gating. This resulted in a dataset of raw images for one cardiac cycle. The raw data was reconstructed in the axial plane, and the cardiac cycle was divided into 10 phases (multiphase image series). Depending on the dimensions of the right ventricle, each cardiac phase consisted of 18-22 axial slices. Each tomogram was $512 \times 512$ pixels in size, with the slice thickness of $0.3-0.6 \mathrm{~mm}$.

Note that in 2D echocardiography we cannot expect the tolerable error to be high due to low accuracy in forming the contours. We assume it is reasonable to keep the error below 15-20\% when estimating the RV's volume. Thus, our aim is to obtain the surfaces satisfying this level of accuracy. However, due to the lack of secure verification criteria, we need several different fitting algorithms to choose from upon cross-validation of the obtained surfaces.

By having three orthogonal flat closed curves "cut out" of the surface, we locate them in 3D space in order to superpose the plane of the curve with the corresponding coordinate plane. So we have a similar situation in each octant: three curves are situated separately in the coordinate planes; each pair of curves has a unique point of intersection on the common coordinate axis.

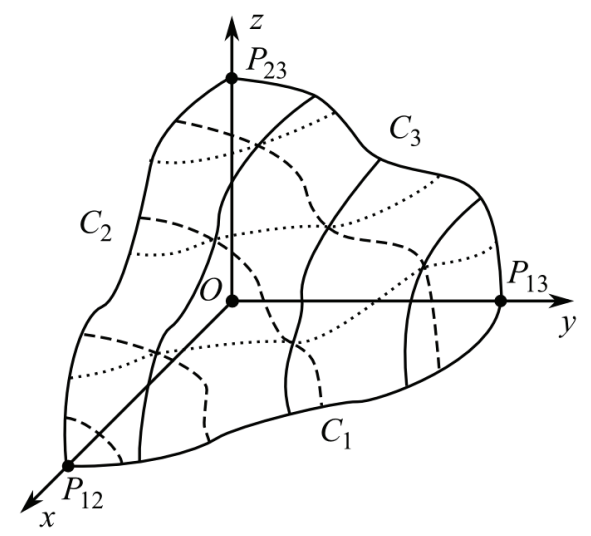

Figure 5. "Pulling" of all 3 contours.

Consider the situation in the first octant (other cases are analyzed in a similar manner). Having three curves $C_{1}, C_{2}$, and $C_{3}$ in the coordinate planes $x O y, x O z$, and $y O z$, respectively, let us denote the points of their pairwise intersections $P_{12}, P_{13}$, and $P_{23}$ (Fig. 5). Assume that locations of two of the curves, e.g., $C_{2}$ and $C_{3}$, are fixed while gradually transferring $C_{1}$ to the point 


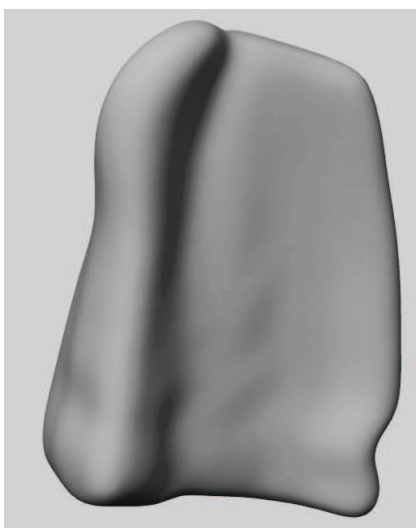

(a)

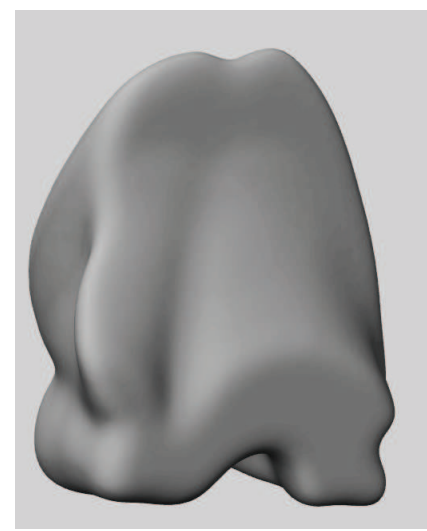

(b)

Figure 6. Two similar surfaces reconstructed from the same set of 3 contours by "pulling" two different contours: along $\mathrm{x}$ axis (a), along y axis (b).

of intersection $P_{23}$ deforming uniformly in order to preserve coincidence of the end points of $C_{1}$ with certain (uniformly spaced) sequence of points on $C_{2}$ and $C_{3}$, respectively.

So we "pull" curve $C_{1}$ from its initial position to the site, where it will contract to the single point $P_{23}$. Hence, we obtain some approximation of the surface that is restricted to the first octant. We then repeat this procedure with other two combinations of curves and declare the weighted sum of three sets of points as a surface restored in this octant (Fig. 6). The choice of the weighting factors was based on the assumption that the factor is inversely proportional to the length of a deformable contour. Weighted factors were varied in our experiments. The surface approximation is represented by a dense grid of triangles.

Applying this algorithm to other octants in a similar way, we can build the final whole surface from 8 pieces. This surface may sometimes require some smoothing operation to achieve a smooth shape. Note that for volume calculation this operation is not necessary.

Smoothing is performed by recursive Gaussian filtering on a discretized representation of the surface. Discretization is performed on a 3D grid, where the inside and outside of the surface gets labeled. The labels are smoothed out, and the final representation of the surface is extracted by the marching cubes or a similar algorithm.

Our algorithm needs no analytical expressions for the curves: all operations are implemented using data set points, which may be considered an advantage.

In our experiments, all the data were derived from $\mathrm{CT}$ scans in an attempt to match the orientations of echocardiography contours as closely as possible. This introduces non-orthogonality in the contour planes. To address this issue, the contours are first transformed in such a way that they intersect with one another, and then new coordinate axes are defined at the points of intersection. This allows obtaining perfectly orthogonal contours. Prior to calculating the 
RV's volume, the final surface is transformed backwards into the initial coordinate space. The error of reconstructed surface volume was $0-5 \%$.To obtain smooth contours from CT data, the data need to be re-sampled and smoothed as described in [21]. Volume is computed by splitting the reconstructed area of the model in elementary tetrahedrons and then computing the sum of all volumes of such elementary tetrahedrons [11].

Table 1. Relative error $\eta$ of computed volume $V_{G}^{A}$ from $2 \mathrm{D}$ echocardiography by using developed model and measured volume $V_{M}$ by using $3 \mathrm{D}$ echocardiography. $V_{G}^{x}$ and $V_{G}^{y}$ are the volumes of generated models.

\begin{tabular}{|c|c|c|c|c|c|}
\hline Ventricle \# & $V_{M}, \mathrm{ml}$ & $V_{G}^{x}, \mathrm{ml}$ & $V_{G}^{y}, \mathrm{ml}$ & $V_{G}^{A}, \mathrm{ml}$ & $\eta, \%$ \\
\hline 1 & 216.6 & 197.96 & 168.36 & 183.16 & $18 \%$ \\
\hline 2 & 101.1 & 125.5 & 102.81 & $114.15 \mathrm{c}$ & $11 \%$ \\
\hline 3 & 154.1 & 128.61 & 146.29 & 137.45 & $12 \%$ \\
\hline 4 & 104.2 & 109.08 & 114.65 & 111.86 & $7 \%$ \\
\hline 5 & 108.8 & 123.46 & 124.03 & 123.75 & $12 \%$ \\
\hline 6 & 64.4 & 79.49 & 61.35 & 70.42 & $9 \%$ \\
\hline 7 & 162.9 & 133.48 & 142.83 & 138.16 & $18 \%$ \\
\hline 8 & 97.7 & 92.37 & 101.01 & 96.69 & $1 \%$ \\
\hline 9 & 206.3 & 286.72 & 202.87 & 244.79 & $16 \%$ \\
\hline 10 & 152.1 & 114.72 & 114.29 & 114.51 & $33 \%$ \\
\hline 11 & 90.4 & 78.51 & 74.41 & 76.46 & $18 \%$ \\
\hline 12 & 162.6 & 155.53 & 125.49 & 140.51 & $16 \%$ \\
\hline 13 & 133.0 & 167.09 & 154.63 & 160.86 & $17 \%$ \\
\hline 14 & 87.0 & 113.64 & 102.63 & 108.13 & $20 \%$ \\
\hline 15 & 150.6 & 194.32 & 169.04 & 181.68 & $17 \%$ \\
\hline 16 & 95.3 & 125.66 & 119.01 & 122.34 & $22 \%$ \\
\hline 17 & 92.4 & 78.34 & 101.18 & 89.76 & $3 \%$ \\
\hline 18 & 57.0 & 62.32 & 78.56 & 70.44 & $19 \%$ \\
\hline
\end{tabular}

\section{Results}

Models of the RV were generated for 18 cases (diastolic and systolic periods) from 54 manually marked contours and echocardiography DICOM images. These were obtained from the patients having various heart diseases like ischemic and valvular heart diseases, myocardial infarction, valvular insufficiency, pulmonary hypertension, cardiomyiopathies. No special requirements were imposed on the human subjects, but CT scans and echocardiography were performed either the same day or in a three days interval in order to avoid anatomic changes. To validate the results, 36 surfaces were generated and then the models computed. Table 1 summarizes relative error of the averaged volume computed from 2D echocardiography by using the proposed model and measured volume by using $3 \mathrm{D}$ echocardiography. The acceptable results show that only 2 models of 18 were computed over $20 \%$ of relative error $\eta=1-V_{M} / V_{G}^{A}$, where $V_{M}$ is a measured volume, $V_{G}^{A}=\left(V_{G}^{x}+V_{G}^{y}\right) / 2 . V_{G}^{x}$ and $V_{G}^{y}$ are the volumes of generated models along $\mathrm{x}$ axis and along y axis. 


\section{Conclusions}

In this paper, we presented a methodology for computing the volume of the human heart's right ventricle using 2D echocardiography images. Upon manually marking three contours of the ventricle's regions on echocardiography DICOM images, these contours are positioned semi-automatically by keeping them orthogonal and concurrent. Then, the ventricle's surface is generated and the volume computed, both operations being fully automated. For each ventricle model, two surfaces were generated and their volumes computed. Final result of the ventricle's volume was calculated as the average of the two computed volumes.

It should be noted that for an investigation aiming to determine merely the volume of the heart's RV, there is actually no need in attempting to restore the RV's surface from the volumetric data (which tends to be rather scarce). In this case, the problem can be approached by estimating the volume in each octant. This requires data for supplementary contours, if available. Our preliminary calculations suggest that this approach may also perform well with a dramatic reduction in computation time.

In case there are more supplementary contours or other additional information about the points of the RV's surface, it can be modeled using partial differential equation (PDE) methods. Then, a boundary value problem for elliptic PDE is solved in each octant, where the function being searched is the distance of the surface points from the origin. Any data available is employed to identify the parameters of the equation. We should be able to perform such computations in the future. $20 \%$ of incorrect volumes clearly evidence a need for a more automatic methodology that is less prone to human error. Also, it is necessary to define the rules for deforming the contours when merging their intersection points. As dictated by the nature of echocardiography, we usually do not have clear boundaries of the ventricle's regions in the planes of interest. In future studies, we shall investigate possibilities for deforming such unclear boundaries for proper positioning of the contours. The proposed method can be used for clinical follow up studies of RV volume changes during the treatment.

\section{Acknowledgment}

This work was supported by the Research Council of Lithuania grant LIG$24 / 2010$.

\section{References}

[1] R.H. Bartels, J.C. Beatty and B.A. Barsky. An Introduction to Splines for Use in Computer Graphics and Geometric Modeling. Morgan Kaufmann, 1987.

[2] E. Catmull and R. Rom. A class of local interpolating splines. In R. Barnhill and R. Riesenfeld(Eds.), Comput. Aided Geom. Design, pp. 317-326. Academic Press, 1974.

[3] Y.-T. Ching, Y.-H. Liu, C.-L. Chang and J.S.J. Chen. Almost automatic method for reconstruction $3 \mathrm{D}$ geometric model of the left ventricle from $3 \mathrm{D}+1 \mathrm{D}$ precor- 
dial echocardiogram. Journal of Medical and Biological Engineering, 21(2):127$133,2001$.

[4] R. Cipolla and A. Blake. Surface shape from the deformation of apparent contours. International Journal of Computer Vision, 9(2):83-112, 1992. http://dx.doi.org/10.1007/BF00129682.

[5] L.L. Creswell, S.G. Wyers, J.S. Pirolo, W.H. Perman, M.W. Vannier and M.K. Pasque. Mathematical modeling of the heart using magnetic resonance imaging. Medical Imaging, IEEE Transactions on, 11(4):581-589, 1992.

[6] VTK User's Guide. Kitware, Inc. 11 edition, 2010.

[7] A.A. Harouni, D.A. Bluemke and N.F. Osman. Fully automated segmentation of long-axis MRI Strain-Encoded (SENC) images using active shape model (ASM). In Biomedical Imaging: From Nano to Macro, 2009. ISBI '09. IEEE International Symposium on, pp. 827-830, 2009.

[8] K.D. Horton, R.W. Meece and J.C. Hill. Assessment of the right ventricle by echocardiography: a primer for cardiac sonographers. J Amer. Soc. Echocardiogr, 22(7):776-92; quiz 861-2, 2009. http://dx.doi.org/10.1016/j.echo.2009.04.027.

[9] L. Ibanez and W. Schroeder. The ITK Software Guide. Kitware, Inc, 2005.

[10] J. Kjaergaard, C.L. Petersen, A. Kjaer, B.K. Schaadt, J.K. Oh and C. Hassager. Evaluation of right ventricular volume and function by $2 \mathrm{D}$ and $3 \mathrm{D}$ echocardiography compared to MRI. EHJ Cardiovascular Imaging, 7(6):430-438, 2006. http://dx.doi.org/10.1016/j.euje.2005.10.009.

[11] E. Kreyszig. Advanced Engineering Mathematics. Wiley, 10 edition, 2011.

[12] R. Kurazume, K. Nakamura, T. Okada, Y. Sato, N. Sugano, T. Koyama, Y. Iwashita and T. Hasegawa. 3D reconstruction of a femoral shape using a parametric model and two 2D fluoroscopic images. In Robotics and Automation, 2007 IEEE International Conference on, pp. 3002-3008, 2007.

[13] R.M. Lang, M. Bierig, R.B. Devereux, F.A. Flachskampf, E. Foster, P.A. Pellikka, M.H. Picard, M.J. Roman, J. Seward, J.S. Shanewise, S.D. Solomon, K.T. Spencer, M.St. John Sutton and W.J. Stewart. Recommendations for chamber quantification: a report from the American Society of Echocardiography's Guidelines and Standards Committee and the Chamber Quantification Writing Group, developed in conjunction with the European Association of Echocardiography, a branch of the European Society of Cardiology. J. Amer. Soc. Echocardiogr, 18(12):1440-1463, 2005. http://dx.doi.org/10.1016/j.echo.2005.10.005.

[14] M. Matačiūnas, D. Zakarkaitè, I. Zeleckienè, D. Palionis, N. Valevičienè, A. Ušinskas and A. Tamošiūnas. Tomographic volumetry of the right ventricle: redundancy of technologies or inescapable necessity for a multimodality approach? Seminars in Cardiovascular Medicine, 17, 2011.

[15] L. Mockus, M. Meilūnas, M. Paulinas, A. Ušinskas and D. Zakarkaitè. Generating of reformat slices in neural and cardio-tomography. Math. Model. Anal., 12(1):121-130, 2007. http://dx.doi.org/10.3846/1392-6292.2007.12.121-130.

[16] S. Nandagopalan, B.S. Adiga, C. Dhanalakshmi and N. Deepak. Automatic segmentation and ventricular border detection of 2D echocardiographic images combining k-means clustering and active contour model. In Computer and Network Technology (ICCNT), 2010 Second International Conference on, pp. 447-451, 2010. 
[17] M. Paulinas. Segmentation and analysis of medical computer tomograms. PhD thesis, Vilnius Gediminas Technical University, Lithuania, 2010.

[18] M. Paulinas and M. Meilūnas. An algorithm for partitioning of right heart ventricle medial axis. Math. Model. Anal., 15(2):245-255, 2010. http://dx.doi.org/10.3846/1392-6292.2010.15.245-255.

[19] S. Prakoonwit and R. Benjamin. 3D surface reconstruction from multiview photographic images using 2D edge contours. 3D Research, 3(4):1-12, 2012.

[20] F. Rademakers. Echocardiographic volumetry of the right ventricle. Eur. J. Echocardiography, 6(1):4-6, 2005. http://dx.doi.org/10.1016/j.euje.2004.11.008.

[21] I.J. Schoenberg. Cardinal Spline Interpolation. SIAM, 1973.

[22] A. Skalski and P. Turcza. Heart segmentation in echo images. Metrology and Measurement Systems, 18(2):305-314, 2011. http://dx.doi.org/10.2478/v10178-011-0012-y.

[23] A. Szymczak, W. Hoff and M. Mahfouz. 3D shape from silhouette points in registered 2D images using conjugate gradient method. Proc. SPIE, 7623:762316762316, 2010.

[24] D. Turco, C. Corsi and C. Lamberti. Fully automated quantification of left and right ventricular volumes throughout the cardiac cycle from magnetic resonance imaging. In Computing in Cardiology, 2011, pp. 377-380, 2011.

[25] A. Usinskas, M. Paulinas, M. Meilūnas, M. Mataciunas, D. Zakarkaite, I. Zeleckiene and A. Laucevicius. Computing of right ventricle volume using orthogonal contours. Electronics and Electrical Engineering, 9:83-86, 2011.

[26] K.-Y. Wong and R. Cipolla. Reconstruction of sculpture from its profiles with unknown camera positions. Image Processing, IEEE Transactions on, 13(3):381389, 2004.

[27] P.A. Yushkevich, J. Piven, H.C. Hazlett, R.G. Smith, S. Ho, J.C. Gee and G. Gerig. User-guided 3D active contour segmentation of anatomical structures: significantly improved efficiency and reliability. Neuroimage, 31(3):1116-1128, 2006. http://dx.doi.org/10.1016/j.neuroimage.2006.01.015.

[28] D. Zhang and X. Wu. An edge-guided image interpolation algorithm via directional filtering and data fusion. Image Processing, IEEE Transactions on, 15(8):2226-2238, 2006.

[29] C. Zhao and R. Mohr. Global three dimensional surface reconstruction from occluding contours. Computer Vision and Image Understanding, 64:62-96, 1994. http://dx.doi.org/10.1006/cviu.1996.0046. 